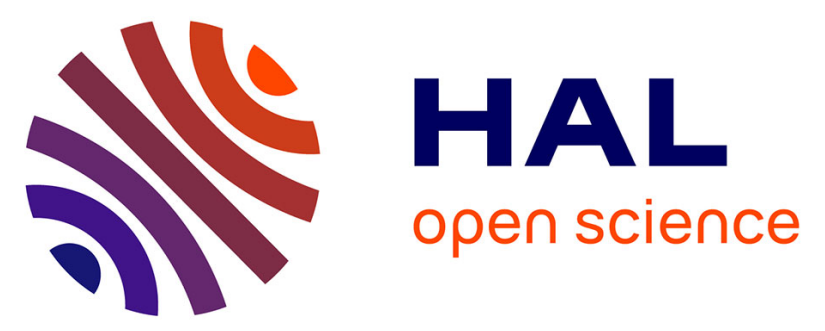

\title{
SEMICLASSICAL AND QUANTUM MECHANICAL TREATMENTS OF POSITIVE AND NEGATIVE ANGLE POLARIZED HEAVY ION SCATTERING
}

D. Mukhopadhyay, G. Grawert

\section{- To cite this version:}

D. Mukhopadhyay, G. Grawert. SEMICLASSICAL AND QUANTUM MECHANICAL TREATMENTS OF POSITIVE AND NEGATIVE ANGLE POLARIZED HEAVY ION SCATTERING. Journal de Physique Colloques, 1984, 45 (C6), pp.C6-435-C6-444. 10.1051/jphyscol:1984652 . jpa00224255

\section{HAL Id: jpa-00224255 https://hal.science/jpa-00224255}

Submitted on 1 Jan 1984

HAL is a multi-disciplinary open access archive for the deposit and dissemination of scientific research documents, whether they are published or not. The documents may come from teaching and research institutions in France or abroad, or from public or private research centers.
L'archive ouverte pluridisciplinaire HAL, est destinée au dépôt et à la diffusion de documents scientifiques de niveau recherche, publiés ou non, émanant des établissements d'enseignement et de recherche français ou étrangers, des laboratoires publics ou privés. 
SEMICLASSICAL AND QUANTUM MECHANICAL TREATMENTS OF POSITIVE AND NEGATIVE ANGLE POLARIZED HEAVY ION SCATTERING

D. Mukhopadhyay and G. Grawert

Institut flir Theoretische Physik, Universität Heidelberg, and

Max-Planck-Institut für Kemphysik, Heidelberg, F.R.G.

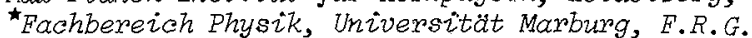

Résumé - Nous utilisons une théorie semiclassique pour calculer les sections efficaces et les pouvoirs d'analyse à tout ordre en diffusion élastique d'ions lourds polarisés. Cette approche a pour but d'expliquer les déviations par rapport à l'absence de structure observée normalement dans les sections efficaces. Ces déviations sont dues aux interférences entre diffraction et réfraction. Nous montrons que la diffusion rëfractive à angle négatif ("farside scattering") peut être interprétée comme un effet de taille de la cible à $E_{1 a b} \approx 20 \mathrm{MeV}$ pour $\overrightarrow{6}_{\mathrm{Li}}+{ }^{12} \mathrm{C}$. Il est montré que le très grand nombre d'intégrales à calculer numériquement limite le champ d'application de la méthode semiclassique. Finalement nous présentons une approche quantique pour traiter la diffusion à angles positifs et négatifs.

Abstract - A unified semiclassical theory for calculating cross section and analysing powers of all ranks for polarized heavy ion elastic scattering is applied to explain deviations from the structureless pattern normally encountered in the cross section, which are interpreted as due to interference between diffraction and refraction. Refractive, negative angle or far-side scattering is then djscussed and is shown to be a target-size effect at $E_{1 a b} \approx 20 \mathrm{MeV}$ for $\overrightarrow{6}_{\mathrm{Li}}+{ }^{12} \mathrm{C}$. The 7 imitations of the semiclassical method are then shown in terms of the necessity of performing a huge number of integrals numericaliy. Lastiy, a quantum mechanical approach for handiing positive and negative angle scattering is presented.

I - INTRODUCTION

Experimental data of polarization observables along with elastic scattering cross sec tions can most assuredly be expected to provide a more stringent handle on studying interference effects arising from negative angle scattering compared to the situation without analysing power measurements. This is because one would then utilise an additional degree of freedom, namely spin, over and above unpolarized cross section measurement, provided one can experimentaliy study effects of spin on cross section, 
i.e. analysing powers. Thus a fuller set of measurements composed of analysing powers of as many ranks as possible for the same scattering process would provide a ground for testing the validity of any model assumptions or resolving of any optical potential ambiguities with much less uncertainty. One such field which has recently received much attention, albeit without accompanying polarization data, is the study of refractive effects in terms of the normal, diffractive positive angie scattering from the near side of the target and the negative angle scattering from the far side. In this talk we present how positive and negative angle scattering and interferences thereof can be theoretically studied using polarization data firstly by adopting a trajectory picture, that is, in a semiclassical manner and secondly by taking the quantum mechanical approach of splitting the scattering process into its near and far-side contributions inasmuch as the Legendre functions, which occur in partia] wave expansion within optical model, are rearranged accordingly.

A series of technical limitations is responsible for the fact that uptil now a complete set of analysing power data is available only for some $\vec{L}_{\mathrm{Li}}$ (spin 1 ) and $\overrightarrow{\mathrm{Li}_{j}}$ (spin $3 / 2$ ) elastic scattering from 1 ight $\left({ }^{12} \mathrm{C}\right)$ and heavier $\left({ }^{58} \mathrm{Ni}\right.$ say) targets at tandem energies. In view of this fact and due to the theoretical considerations introduced above, in this work we concentrate on developing a theoretical approach which is not only capable of simultaneously accounting for the observed cross section as well as analysing powers of all ranks, but also can incorporate a specific effect arising out of the size of the target.

To understand the target-size effect, we present the following argument for visualising elastic scattering of a light heavy-ion (HI) projectile like the lithium isotopes. Positive angle scattering is then the dominant process for scattering from a heavy target (say ${ }^{58_{\mathrm{Ni}}}$ or heavier) since the negative angle contributions are heavily suppressed as projectiles which go around the target get more strongly absorbed. This is how we explain the relatively featureless Fresnel-type diffraction pattern for $\sigma / \sigma_{R}$ of $\overrightarrow{\mathrm{Li}}+{ }^{58_{\mathrm{Ni}}}$ elastic scattering at $\mathrm{E}_{1 \mathrm{ab}}=20.3 \mathrm{MeV}[1]$ and the corresponding second rank tensor analysing power $\mathrm{T}_{20}$ is then well explained by taking recourse to the shape effect model [2]. This classical model associates changes in overlap between projectile and target at the point of closest approach to aligning quadrupoledeformed projectiles (here ${ }^{7} \mathrm{Li}$ with $\mathrm{Q}$-moment $=-3.9 \mathrm{fm}^{2}$ ) with respect to various spin projections, since in a spin-polarized beam quadrupole moment and spin are coaxial. Thus the shape-effect model not only gives the correct (negative) sign of $\mathrm{T}_{T_{20}}$ for $\overrightarrow{7}_{\mathrm{Li}}+58_{\mathrm{Ni}}$ beyond the grazing angle but also reproduces the magnitude for a reasonable choice of ${ }^{7} \mathrm{Li}$ quadrupole moment.

In contrast, for elastic scattering of lithium with the lighter target ${ }^{12} \mathrm{C}$ at comparable laboratory energies [3], the angular distribution of $\sigma / \sigma_{R}$ shows wiggles for angles beyond the grazing angle and the simple shape effect model for obtaining the 
analysing powers is automatically doomed to failure. Here, one can argue that the wiggles are due to interference effects between diffraction and negative angle scattering and therefore the simplified version of the shape effect model can be bidden good-bye. In this work we show how the 'nuclear matter overlap' ideology can be extended in a heavily modified form to negative angle scattering. Here we again reiterate the notion that treating $\sigma / \sigma_{R}$ and ${ }^{T_{T}} T_{20}$ on the same footing provides an extremely powerful methodology of obtaining a handle on negative angle scattering.

This report is divided into four sections. Polarization observables are briefly introduced and defined in sect. 2. Then we summarily discuss the semiclassical version of treating negative angle scattering in sect. 3 where the reader will be referred to our work on a general formalism of semiclassical theory of analysing powers. Next, in sect. 4 the quantum mechanical approach of separating the scattering process into positive and negative angle contributions is dealt with.

\section{II - DEFINITION OF ANALYSING POWERS}

Starting with the Schroedinger equation $H \psi=E \psi$, the scattering amplitude matrix $M$ is defined by the asymptotic expansion of the total wave function $\Psi$ as a sum of an incoming plane wave in a pure spin state $m_{0}$ and an outgoing spherical wave composed of all possible spin states. Thus

$$
\psi \underset{r \rightarrow \infty}{\longrightarrow} e^{i \vec{k} \vec{r}}\left|m_{0}>+\frac{e^{i k r}}{r} \sum_{m}\right| m>\left\langle m|M| m_{0}>\right.
$$

where $k$ is the wave number.

The centre-of-mass (c.m.) cross section $\frac{d \sigma}{d \Omega}\left(\theta_{c . m .}, \rho_{\text {in }}\right)$ is obtained by transforming from the measured laboratory angle $\theta_{1 a b}$ to the corresponding c.m. angle. Thus,

$$
\frac{d \sigma}{d \Omega}\left(\theta_{c, m}, \rho_{i n}\right)=\operatorname{Tr} M^{+} \rho_{\text {in }} M=\operatorname{Tr} \rho_{\text {in }} M^{+} M
$$

where the spin state of the beam is described by the density matrix $\rho_{\text {in }}$ in spin space.

Expanding $\rho_{\text {in }}=\dot{0} \leqslant k \leqslant 2 s \tau_{\mathrm{kq}} t_{\mathrm{kq}}^{*} /(2 s+1)$, where $s$ is the projectile spin, $k$ and $q$ are the rank of the tensor and $i$ ts projection and $\left\langle m^{\prime}\left|\tau_{k q}\right| m\right\rangle=\sqrt{2 k+1} c$ (sks; mqm') are the irreducible spherical matrices in spin space, one obtains

$$
\begin{aligned}
& \frac{d \sigma}{d \Omega}\left(\theta_{c . m .}, \rho_{i n}\right)=\frac{1}{2 s+1} \Sigma t_{k q}^{*} T r \\
& k q^{M} M=\frac{\operatorname{Tr} M^{+} M}{2 s+1}\left\{1+\sum_{k>1} t_{k q}^{*} T_{k q}\right\} . \\
& \text { Here, } \frac{T r M^{+} M}{2 s+1} \equiv\left(\frac{d \sigma}{d \Omega}\right)_{\text {unpol. }} .
\end{aligned}
$$


The $t_{\mathrm{kq}}$ 's denote the 'prepared' beam polarization and the $T_{\mathrm{kq}}$ 's are the measured analysing powers.

\section{Specialisation}

Rank $1(k=1)$ : For the rank 1 case the beam is 'prepared' in a way such that the matrix $\rho$ has the following elements:

$$
<m^{\prime}\left|\rho_{i n}\right| m>=\delta_{m m^{\prime}} \frac{1+m / s}{2 s+1},
$$

that is, the occupation number is a linear function of the magnetic substates. Clearly then, $p_{i n}=\frac{1}{2 s+1}\left\{1+\frac{1}{s} \vec{s} . \hat{e}\right\}$ and in this construction the only non-zero $t_{1 q}$ is $t_{10}$.

Such a beam is called a 'vector polarized' beam. If now one measures the transverse vector (or first rank) analysing power, called ${ }^{\mathrm{T}} \mathrm{T}_{10}$, by taking the quantisation axis such that $\vec{e} \| \vec{p}_{j} \times \vec{p}_{f}$, then this 'transverse' quantity is

$$
T_{T_{10}}=\frac{1}{t_{10}} \frac{\left(\frac{d \sigma}{d \Omega}\right)_{p o l}-\left(\frac{d \sigma}{d \Omega}\right)_{\text {unpol. }}}{\left(\frac{d \sigma}{d \Omega}\right)_{\text {unpol. }}} .
$$

For the transversity frame the choice of axis is $\vec{p}_{i} \times \vec{p}_{f} \| \vec{e}_{z}$ and for the Madison frame [4] one chooses $\vec{p}_{i} \times \vec{p}_{f} \| \vec{e}_{j}$. For rank 1 the 'transverse' $T_{10}$ is simply proportional to 'Madison' $i T_{11}$ (as a whole a real quantity!), i.e., $T_{T} T_{10}$ a $i M_{11}$. Further details on this subject and interrelations among the various conventions of defining polarization observables can be obtained from ref. [4].

Rank $2(k=1)$ : Here we shall simplify the discussion by directly considering the situation for spin $3 / 2$. In this case the beam is 'prepared' such that only $t_{20}=1$ and all other $t_{\mathrm{kq}}$ 's are zero. This is achieved, say, by having a quadratic (parabolic) behaviour of the occupation number as a function of the magnetic projection as follows:

$$
<m^{\prime}\left|\rho_{\text {in }}\right| m>= \begin{cases}\frac{1}{2} & \text { for } m^{\prime}=m=3 / 2 \\ 0 & \text { otherwise }\end{cases}
$$

Such a beam is called 'aligned' and the transverse tensor (or second rank) analysing power is

$$
T_{T_{20}}=\frac{\left(\frac{d \sigma}{d \Omega}\right)_{a l i g n e d}-\left(\frac{d \sigma}{d \Omega}\right)_{\text {unpol. }}}{\left(\frac{d \sigma}{d \Omega}\right)_{\text {unpol. }}} .
$$




\section{II - SEMICLASSICAL TREATMENT OF POSITIVE AND NEGATIVE ANGLE SCATTERING}

In the following we only shall briefly indicate those steps in the formalism of treating negative angle scattering essential for our calculations. Detailed treatment of positive angle scattering and polarization observables within the semiclassical framework is given in our paper, vide ref. [5].

We start with taking the real central potential $v_{c}$ to be the dominant interaction and treat $v_{c}$ in a classical approximation to obtain trajectories of the projectile. All the rest of the potentials, be they absorptive central $\left(w_{c}\right)$ or complex spin-orbit $(U)$ are treated perturbatively. The absorption, governed by $w_{c}$, therefore, takes place simply while the projectile moves on its trajectory and the spin-dependent interactions, caused by $U$, generate a time-evolution of the projectile spin state again without affecting the trajectories. As a sequel to these assumptions the S-matrix factorizes into a product of two terms, the first factor being the S-matrix for central potentials and the other being a matrix $B$ in spin space, which narrates the effects of spin-dependent interactions on the spin state of the projectile. Next, the S-matrix so obtained is inserted into a semiclassical expression [6] for the matrix $M$ of scattering amplitudes. The usual partial wave sum for the scattering amplitude is transformed into an integration over trajectories and the M-matrix is evaluated by the method of stationary phases [7]. Finaliy, from the M-matrix the analysing power for elastic polarized HI scattering are calculated applying standard definitions [4] used for describing polarization phenomena.

Starting with the full time-dependent Schroedinger equation for the relative motion of particles with a real central interaction, we make the following simplifications as regards the trajectories. Firstly, the wave packet is a narrow one and secondly, its centre moves along the trajectory. The Morse saddle point equations for absolute values of $L$ are

$$
\frac{\partial}{\partial L}{ }^{2 \delta_{C}}(L)+\varepsilon \theta+2 \pi \nu=0 \quad(\varepsilon= \pm 1)
$$

and the general behaviour of the classical deflexion function

$$
\theta_{c}(L)=\partial 2 \delta_{c}(L) / \partial L
$$

is sketched in fig. 1 of ref. [5], where it can be seen that $\theta_{c}$ is determined essentially by coulomb repulsion for large $L$ values and it goes through the rainbow angle $\theta_{R}$ at $L=L_{R}$ and becomes infinite for grazing angular momentum $L_{g}$. In table 1 of ref. [5] the rainbow parameters are presented for the currently available data sets for the two projectiles $\overrightarrow{\sigma_{L i}}$ and $\overrightarrow{L i}$. 
For the sake of methodological consistency within the semiclassical framework, $\delta_{c}$, the real central phase shift is taken to be the WKB one corresponding to the real central potential $v_{c}$ and the central absorptive coefficient, $\alpha_{c}$, is defined by

$$
\alpha_{c}(L)=-\frac{1}{h} \int_{-\infty}^{\infty} w_{c}\left(R_{t}\right) d t \text {. }
$$

Two graphical solutions of eq. (1) for a specific $\theta>{ }_{R}$ are also depicted in fig. 1 of ref. [5]. If we have $\theta<\theta_{R}$, there exist also two solutions of eq. (1) for $v=0$, $\varepsilon=-1$, i.e., for positive deflexion angles $\theta$. These last two solutions become complex when varying $\theta$ from values below $\theta_{R}$ to those above of it. As in ref. [8], approximate complex solutions are calculated by expanding $\theta_{C}$ around $L_{R}$ and solving

$$
\theta_{R}+\frac{1}{2}\left(L-L_{R}\right)^{2} \theta_{R}^{\prime \prime}-\theta=0,
$$

$\theta_{R}^{\prime \prime}$ being the second derivative of $\theta_{C}$ at $L=L_{R}$. Thus, for $\theta<\theta_{R}$, $L_{0}$ which represents actually two values of angular momenta, one on the ascending and the other on the descending side of the deflexion function, corresponds to a pair of positive angle contributions denoted by $g_{0}$. Also for $\theta<\theta_{R}, L_{1}$ lying as it does between 0 and $-\pi$ generates the negative angle contribution $g_{1}$ and $L_{2}$ lying between $-\pi$ and $-2 \pi$ gives the negative angle contribution for a single orbiting around the target called $\mathrm{g}_{2}$ etc. For $\theta>\theta_{R}$ one of the pair of complex solutions $L_{0}$ must be taken into consideration aside from the real solutions of eq. (1), i.e., $L_{1}, L_{2}$ etc. The other complex solution is to be discarded because this would have an exponentially increasing contribution to the scattering amplitude. In other words, for a Fresnel diffraction pattern of the cross section, the diffraction amplitude $g_{0}$ dominates and if one has a wiggly $\sigma / \sigma_{R}$, this is caused by interference between $g_{0}$ and $g_{1}$.

The next step consists of introducing spin-dependent potentials into the above-discussed procedure for central potentials and writing down the time-dependent Schroedinger equation for the spin-state evolution matrix $B$. This generates spin-orbit phase shifts $\delta_{\text {so }}$ given by

$$
2 \delta_{\text {so }}(L)=-\frac{L}{\hbar} \int_{-\infty}^{\infty} v_{\text {so }}\left(R_{t}\right) d t \text {. }
$$

The physical meaning of this result is that projectiles in particular eigenstate of $\overrightarrow{\mathrm{s}} \cdot \overrightarrow{\mathrm{L}}$ will stay in the same one while moving along a trajectory. The real spin-orbit phase shift $2 m \delta_{\text {so }}$ and the spin-orbit absorption coefficient $\alpha_{\text {so }}$, for $w_{\text {so }} \neq 0$, depend on $m$, the eigenvalue of $\vec{s} \cdot \vec{L}$. Needless to say that the introduction of spin-dependent potentials modifies the saddle point equation (1) accordingly, encompassing spin-dependent forces and spin-flip processes. As a result of the factorization property mentioned above, we now have, associated with each $g_{j}$, a corresponding multiplicative factor $h_{j}$ due to the spin-dependent potentials. 
When diffraction scattering dominates, say, for a heavy target, i.e., $\sigma / \sigma_{R}$ shows Fresnel pattern, we know from above that then only $g_{0}$ contributes to $f_{c}$. Calculations were done for ${ }^{i T_{11}}$ for such a situation and the result is shown in fig. 4 of ref. [5]. The agreement with data is excellent inasmuch as the calculation is parameterfree.

We now present the theory for calculating $g_{1}$, i.e., consider a scattering process like. $\overrightarrow{{ }_{L i}}+{ }^{12} \mathrm{C}$ where the diffraction term plus non-orbiting negative angle term and interferences thereof all play a role due to the smaller target size. The input potentials [3] obtained by optical model fit are the real and the imaginary central potentials and the real and the imaginary spin-orbit potentials.

The procedure is divided into three steps.

a) As for the positive angle case, one determines the deflexion function $\theta$ and obtains therefrom the rainbow parameters $\theta_{R}, L_{R}$ and $\theta_{R}^{\prime \prime}$, where the double dash indicates the second derivative with respect to $L$ and the corresponding rainbow absorption coefficients $\alpha_{C}\left(L_{R}\right)$ and $\alpha_{\text {so }}\left(L_{R}\right)$ and the rainbow spin-orbit phase shift ${ }^{2 \delta_{S O}}\left(L_{R}\right)$ and its derivative $2 \delta_{\text {so }}$ '.

b) Then one determines the range $R$ of $R_{0}$ values, $R_{0}$ being the distance of closest approach, for which $-\pi \leqslant \theta \leqslant 0$, i.e., one is in the negative angie range.

c) Divide the range $R$ into parts so that oscillations in the experimental data can be reproduced. Note that the relationship between $R_{0}$ and $\theta_{c . m}$. is not linear. Concretely speaking, if experimental data exist between, say, $30^{\circ}$ and $130^{\circ}$, i.e., within an angular range of $100^{\circ}$ and oscillations need to be reproduced to within $0.5^{\circ}$, one then divides $R$ into approximately 200 parts, taking care of sight nonlinearity. Then, for each of these values of $R_{0}$, one calculates $L$ and $\theta$ and the absorption coefficients $\alpha_{c}$ and $\alpha_{\text {so }}$ along with $2 \delta_{\text {so. }}$. In addition, the following integral is needed, namely,

$$
x=-\int_{L}^{L} \theta\left(L^{\prime}\right) d L^{\prime}
$$

and the derivatives $\theta^{\prime}, \alpha_{c}{ }^{\prime}, 2 \delta_{\text {so }}{ }^{\prime}, \theta^{\prime \prime}$ and $2 \delta_{\text {so }}{ }^{\prime \prime}$ with respect to $\mathrm{L}$.

After having numerically obtained these many quantities, we can now write down the last set of formulae to obtain $\sigma / \sigma_{R}$ and $i T_{11}$. Thus, the diffraction and the nonorbiting negative angle amplitudes are, respectively,

$$
\left|g_{0}\right|=\sqrt{2 \pi L_{R}}\left[\frac{2}{\left|\theta_{R}^{\prime \prime}\right|}\right]^{1 / 3}|A i(x)| e^{-\alpha_{C}\left(L_{R}\right)} \text { with } x=\left[\frac{2}{\left|\theta_{R}{ }^{\prime \prime}\right|}\right]^{1 / 3}\left(|\theta|-\theta_{R}\right)
$$

and $\left|g_{1}\right|=\sqrt{\frac{L}{\theta^{\prime}}} e^{-\alpha} c$, Ai being the Airy function, and the phase 


$$
\psi=x+\left(L+L_{R}\right)|\theta|-\frac{\pi}{4}+\frac{\pi}{2}(1-\operatorname{Sign} A i(x)) .
$$

The corresponding spin-dependent functions, $h_{j}$, are

$$
\begin{aligned}
& h_{0}=-2 \delta_{S O}{ }^{\prime}\left(L_{R}\right)\left[\frac{2}{\mid \theta_{R}{ }^{\prime \prime}}\right]^{1 / 3} \cdot \frac{A i^{\prime}(x)}{A i(x)} \\
& h_{1}=-\frac{2 \delta_{S O}^{\prime}}{2 L \theta^{\prime}}+\frac{\theta^{\prime \prime} 2 \delta_{S O}{ }^{\prime}}{2\left(\theta^{\prime}\right)^{2}}-\frac{2 \delta_{S O}^{\prime \prime}}{2 \theta^{\prime}}+\frac{\alpha_{c^{2}} \delta_{S O^{\prime}}}{\theta^{\prime}} .
\end{aligned}
$$

Thus, the unpolarized cross section is

$$
\frac{\sigma}{\sigma_{R}}(\theta)=\frac{8 E(\hbar c)^{2}}{m_{r e d}\left(z_{1} z_{2} e^{2}\right)^{2}} \cdot \frac{\sin ^{4}\left(\frac{\theta}{2}\right)}{\sin |\theta|} F_{0}
$$

and the first rank vector analysing power is

$$
i T_{11}(\theta)=\frac{F_{1}}{F_{0}} 2 \sqrt{\frac{s(s+1)}{6}}
$$

with $F_{0}=\left|g_{0}\right|^{2}+\left|g_{1}\right|^{2}+2\left|g_{0}\right|\left|g_{1}\right| \cos \psi$

$$
\text { and } \quad \begin{aligned}
F_{1}= & -\left\{\left|g_{0}\right|^{2}+\left|g_{0}\right|\left|g_{1}\right| \cos \psi\right\}\left\{h_{0}-\alpha_{s o}\left(L_{R}\right)\right\} \\
& +\left\{\left|g_{1}\right|^{2}+\left|g_{0}\right|\left|g_{1}\right| \cos \psi\right\}\left\{h_{1}-\alpha_{s o}\right\} \\
& -\left|g_{0}\right|\left|g_{1}\right| \sin \psi\left\{2 \delta_{s o}+2 \delta_{s o}\left(L_{R}\right)\right\}
\end{aligned}
$$

For each of the numerous $R_{0}$ values, one thus has to calculate several integrals and differential coefficients along the trajectory. In this process, extreme care has to be given to avoid convergence problems, particularly for integrals near $R_{0}$. Computational work is in progress and the typical time for one full calculation is about $10 \mathrm{CPU}$ minutes on a CRAY computer. This clearly demonstrates that one has reached the limit of viability of such semiclassical calculations numerically and therefore in the next section we shall present how to go about tackling this problem by a method which should be more suited for it, namely the quantum mechanical procedure.

\section{IV - QUANTUM APPROACH TO POSITIVE AND NEGATIVE ANGLE SCATTERING}

In order to simplify the discussion, we consider for the time being only central potentials. Starting from the partial wave sum for the scattering amplitude 


$$
f(\theta)=\frac{1}{\operatorname{2ik}} \sum_{l=0}^{\infty} P_{1}(\cos \theta)\left(s_{1}-1\right)(21+1),
$$

we decompose the Legendre polynomials as follows

$$
P_{1}=\frac{1}{2}\left(P_{1}+i \frac{2}{\pi} Q_{1}\right)+\frac{1}{2}\left(P_{1}-i \frac{2}{\pi} Q_{1}\right)
$$

where $Q_{1}$ are the Legendre functions of the second kind $[9]$.

$$
\text { Put } \begin{aligned}
Q_{1}{ }^{(-)} & =\frac{1}{2}\left(P_{1}+i \frac{2}{\pi} Q_{1}\right) \\
Q_{1}{ }^{(+)} & =\frac{1}{2}\left(P_{1}-i \frac{2}{\pi} Q_{1}\right) .
\end{aligned}
$$

Then, $f(\theta)=f^{(-)}+f^{(+)}$

with $f^{( \pm)}=\frac{1}{2 i k} \sum_{l=0}^{\infty} Q_{1}^{( \pm)}\left(s_{1}-1\right)(21+1)$

and

$$
\frac{\sigma}{\sigma_{R}} \equiv|f|^{2}=\left|f^{(-)}\right|^{2}+\left|f^{(+)}\right|^{2}+\text { interference terms. }
$$

Semiclassically, the amplitude $f^{(-)}$corresponds to the diffractive/positive angle contribution and $f^{(t)}$ to the refractive/negative angle contribution.

Satchler and coworkers [10] have coined the phrase 'near-side' and 'far-side'for the two cases respectively. This association is obvious, since

$$
Q_{1}^{( \pm)} \simeq \frac{e^{i\left\{ \pm i\left(1+\frac{1}{2}\right) \theta-i \frac{\pi}{4}\right\}}}{\sqrt{2 \pi\left(1+\frac{1}{2}\right) \sin \theta}} \quad \text { for large } 1
$$

The extension to include spin-dependent potentials is relatively straightforward, albeit cumbersome. For example, we now need the associated Legendre functions of the second kind instead of the ordinary ones since magnetic projection is of paramount importance now. In particular, we note that in the case of $\mathrm{Li}+\mathrm{Ni}$ at $\mathrm{E}_{\mathrm{lab}} \leqslant 20 \mathrm{MeV}$ scattering is essentially near-side. Contrarily, in the case of $\frac{L i}{7}+C$ both near-side and far-side amplitudes contribute and interfere. Clearly, the $\overrightarrow{\bar{L}_{i}-T_{2 q}}$ near-side can be expected to behave as the shape effect model predicts, while the far-side $T_{2 q}{ }^{\prime} s$ do not. Work is in progress to introduce these changes into the spin $3 / 2$ optical model code LINA. 


\section{REFERENCES}

[1] FICK, D., Ann. Rev. Nucl. Sci. 31 (1981) 53

[2] ZUPRANSKI, P., DREVES, W., EGELHOF, P., MOEBIUS, K.-H., STEFFENS, E., TUNGATE, G. and FICK, D., Phys. Lett. $91 B$ (1980) 358;

MOROZ, Z., ZUPRANSKI, P., BOETTGER, R., EGELHOF, P., MOEBIUS, K.-H., TUNGATE, G., STEFFENS, E., DREVES, W., KOENIG, I., and FICK, D., NuCl. Phys. A381 (1982) 294

[3] RUSEK, K., MOROZ, Z., CAPLAR, R., EGELHOF, P., MOEBIUS, K.-H., STEFFENS, E., KDENIG, I., WELLER, A, and FICK, D., Nucl. Phys. A407 (1983) 208

[4] SIMONIUS, M. in Lecture notes in physics, vol. 30, ed. FICK, D. (Springer, BerIin, 1974) p. 38

[5] GRAWERT, G. and MUKHOPADHYAY, D., NUC1. Phys. A415 (1984) 304

[6] CROWLEY, B.J.B, and HILL, T.F., Z, Phys. A300 (1981) 299

[7] FORD, K.W. and WHEELER, J.A., Ann. of Phys. 7 (1959) 259

[8] BROGLIA, R.A. and WINTHER, A., Heavy ion reactions, v01. 1 (Benjamin Cummings, London, 1981)

[9] ABRAMOWITZ, M. and STEGUN, I.A., Handbook of mathematical functions (Dover, New York)

[10] SATCHLER, G.R., FULMER, C.B., AUBLE, R.L., BALL, J.B., BERTRAND, F.E., ERB, K.A., GROSS, E.E. and HENSLEY, D.C., Phys. Lett. 128B (1983) 147 